\title{
AN ELECTROSTATIC VOLTMETER
}

\author{
By Warren W. Nicholas
}

ABSTRACT

An electrostatic voltmeter possessing several unique features is described. The size is a minimum for such instruments, since the potential difference is applied to two electrodes, the geometrical arrangement of which is analogous to concentric spheres. The inner electrode is supported on the end of an insulating bushing introduced through the outer electrode, which is a metal can. The inner electrode contains a suspended dumb-bell rotated by electrostatic repulsion, as in the Coulomb torsion balance. The deflection is read by means of a mirror, which, for convenience in mounting the instrument at a safe height, may be placed at the lower end of the insulating bushing. For purposes of automatic control, the dumb-bell may be connected mechanically with an apparatus at ground potential. The design may be adapted to the use of high gas pressures, desirable in the measurement of very high voltages. The sensitivity of the instrument is discussed and calibration curves given for various suspensions.

So many and such varied types of electrostatic voltmeters are described in the literature that the value of publishing a new design may well be questioned. However, it is a matter of common observation that the design for a simpler, less expensive, or more easily constructed instrument is always of considerable interest to investigators; this is probably chiefly due to the fact that the average worker spends a considerable share of his time and energy trying to improve the design of his apparatus. Accordingly, an electrostatic voltmeter is here described which seems to possess several points of unique interest. The writer has used instruments of this design for several years with entire satisfaction. Many of the details are not original, the ideas being accumulated in the X-ray laboratories at Stanford, Cornell, and Columbia.

In Figure 1 is shown a vertical section through the instrument. A circular metal base $B$ is supported on three insulators $A$ by means of leveling screws. Resting on $B$ and inclosing the system $C$ is an inverted metal can $H$ which protects $C$ from dust and air currents and fixes the electrical characteristics of the inside of the instrument (that is, if the can were omitted, the electrical fields near $C$ would depend to some extent on the distribution of charge over the walls of the room and the furniture, and this distribution would be expected to vary with weather conditions and arrangement of furniture). Through the center of $B$ passes an insulating bushing $I$ which supports the moving system. This latter is hung from the suspension $S$, and consists of a dumb-bell $K$, a stiff wire $W$ passing down through the center of the insulating bushing, a disk $V$ immersed in a light oil for damping, and a mirror $M$, by means of which the displacement of the moving system may be read optically. The moving system should be of reasonably light weight and small moment of inertia in order to reduce strains on the suspension, and to minimize the period of oscillation.

$$
89194-31-8
$$


In Figure 2 are shown a vertical section (above) and a horizontal section (below) through $C$. To the base $F$ are fastened the cylinders $L$; the deflection of the moving system is due to the electrostatic repulsion between $L$ and $K$. The mechanism at $D$ provides a means for adjusting the angle $\theta_{0}$ at which the dumb-bell is permanently set (position for zero applied voltage). The various parts of $C$ are designed so as to eliminate all sharp corners and edges from which brush discharge might take place; to this end both the suspension $S$ and the part of the wire $W$ which projects above $F$ are inclosed in

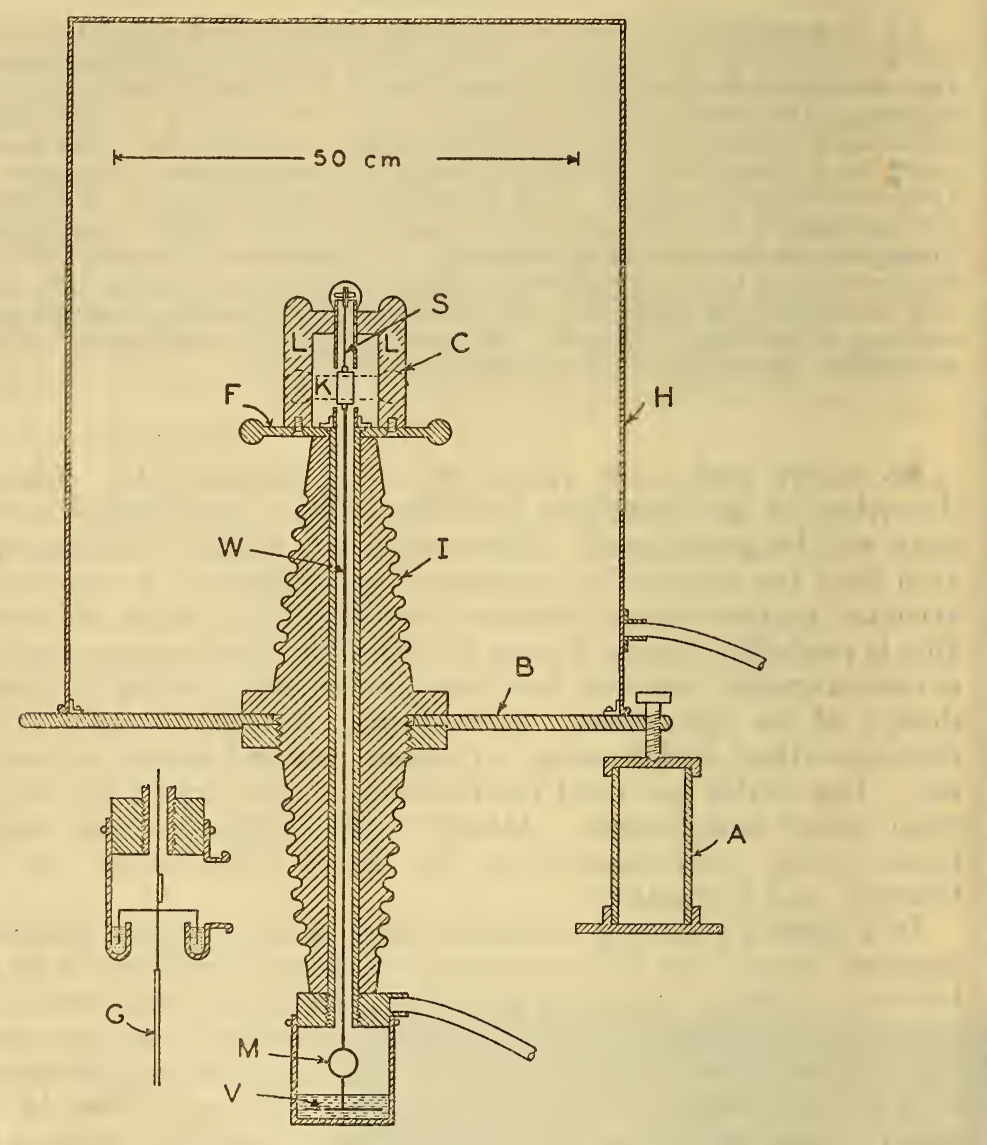

FIGURE 1.-Vertical section through voltmeter

tubes $T$ and $U$, and $D$ is covered with a rounded cap. So far as possible all insulating materials inside the can $\mathrm{H}$ must be electrically shielded from the repulsion system $K$ and $L$, for the variation of the potential of a surface not thus shielded would vary the electrical fields near $K$ and $L$, and consequently the deflection. A slight amount of brush discharge (or perhaps even the residual ionization) might conceivably cause such variations of potential on a surface of excellent insulating properties, but probably the most noticeable effect would be found when the voltage on the instrument was changed. Such a change requires a readjustment of surface charges throughout 
the instrument, and this readjustment would require a period of time in the case of an insulating surface. ${ }^{1}$
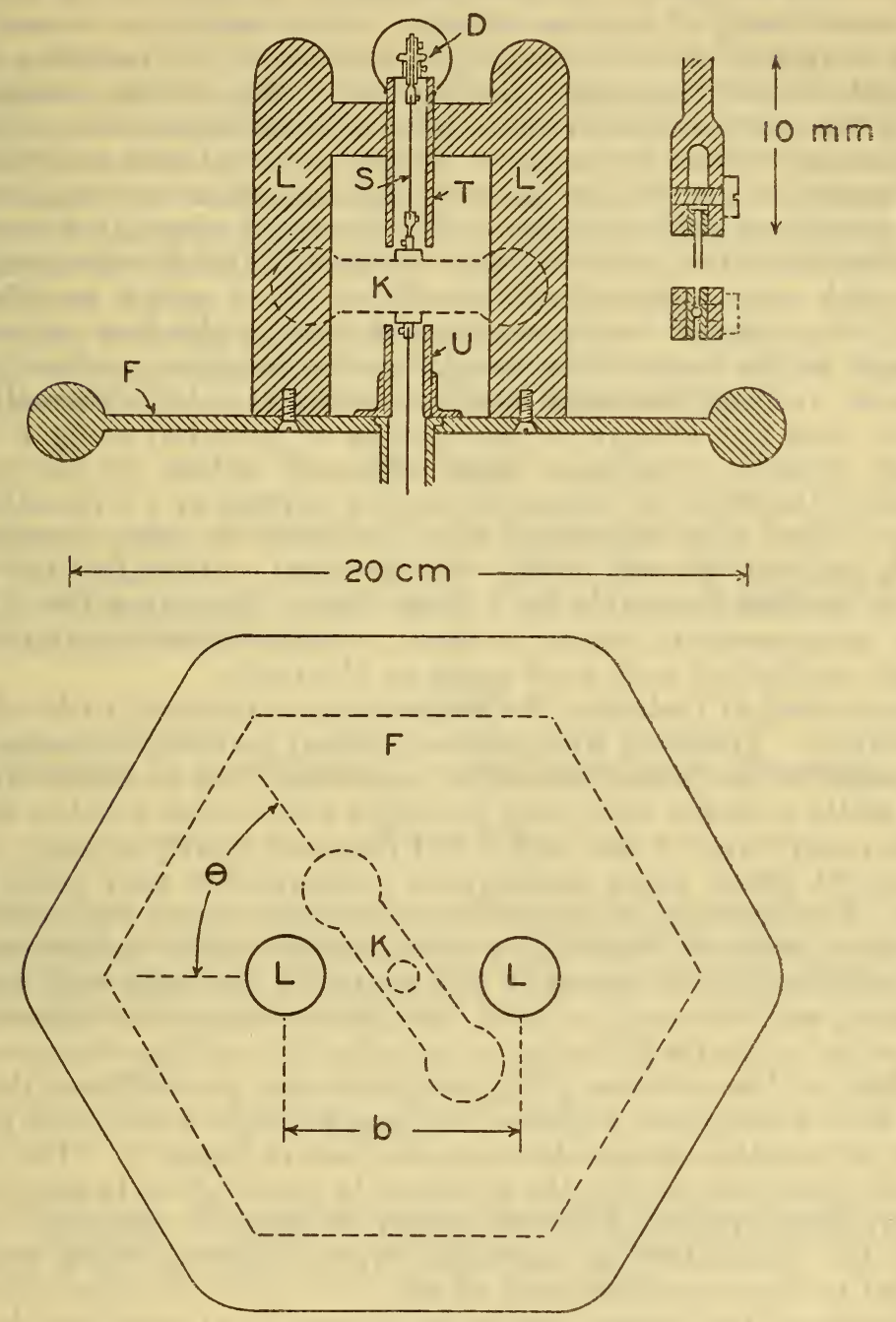

FIGURE 2.-Upper figure, vertical section. Lower figure, horizontal section

The presence of part of the insulating bushing $I$ inside of $H$ can not be avoided, of course; in order to shield $K$ and $L$ from the electric

1 The writer bas experienced analogous difficulties in connection with the use of a galvanometer in the high tension circuit (usually at the cathode potential) for the purpose of measuring the cathode ray current in $\mathrm{X}$-ray tubes. The galvanometer was of the wall type, having a wooden back and a large glass front both very close to the moving coil. Whenever the cathode potential was varied the deflection would he markedly disturbed, but would drift slowly back to the correct $\nabla$ alue. The disturbances were prevented by electrical shielding of the moving system from insulated surfaces. Galvanometers similar to Leeds \& Northrup type $R$ have not been shown to be appreciably subject to such disturbances, but nevertheless even when instruments of this design are used by the writer in a high-voltage system, the glass front is shielded from the moving coil (except for a small hole in front of the mirror) and also a bent strip of sheet metal is slipped down into the hole containing the lower suspension and connected electrically to the magnets or case. In some galvonometers the case itself is found to be insulated from the electrical circuit; this condition should be remedied, 
fields due to the distribution of potential along the surface of $I$, the base $F$ is extended well beyond the cylinders $L$. The question may well be raised whether the distribution of potential along $I$ always duplicates exactly at a given voltage. For example, it is conceivable that a variation of humidity might cause a slight variation of the potential distribution along the insulator; this would change very slightly the electric fields in the vicinity of $K$ and $L$ and cause a change in the amount of the deflection. It was expected that such disturbances would, in general, be quite negligible, but an experimental test of the point was made as follows: The part of the insulator extending from the base $B$ to a point halfway between $B$ and $F$ was wound with wire which was connected electrically to $B$. A rough recalibration up to $25 \mathrm{kv}$. (higher voltages were not used in this test on account of danger to the insulator) showed that the increase produced in the voltmeter reading was only about 1 per cent. It is scarcely conceivable that any change in distribution of potential along $I$ aue to varying climatic conditions could approach within 10 per cent of producing the effect of making so large a portion of $I$ a metallic conductor. Thus it is estimated that the effect of such disturbances will, in general, be well within 0.1 per cent. Actually, the errors may be smaller than this by a large factor, but since few, if any, $\mathrm{X}$-ray measurements require a more accurate determination of the voltage, no further tests were made on the point.

The method of fastening the suspensions is believed to be of some importance. Tungsten wire makes excellent material for suspensions on account of its favorable elastic properties, but to avoid crushing such brittle material care must be taken not to clamp it too tightly. On the other hand, if the wire is not clamped tightly enough, and at the correct point, some undesirable consequences may conceivably occur. For example, suppose that the extreme upper end of the wire is clamped securely, but that a point slightly lower is fastened just insecurely enough to permit it to turn in its fastening with friction. The effect will obviously be that, at a given voltage, the deflection of the moving system will depend on whether this voltage is approached from high or low voltages. To minimize such possibilities, the ends of the wire were placed between thin copper strips which were pressed tightly in the steel clamps shown in the inset of Figure 2. The copper was soft enough to permit the tungsten to press into it to some extent without being crushed, but stiff enough to clamp it securely. In the use of the voltmeter, no anomalies were observed which could be ascribed to the above frictional effects.

A feature of the present design which may be of considerable value for some purposes is that the deflection of the moving system may be readily transmitted mechanically to an apparatus electrically grounded. In the inset of Figure 1 is indicated a means for doing this. By a slight modification of the construction of the damping disk and oil cup, the lower end of the moving system may be attached to a light glass rod $G$, the lower end of which may be at low voltage. By this means, for example, a sensitive relay system could be operated so as to provide a rough control of the voltage. Or a device could be readily constructed by means of which the power would be cut off if the voltage exceeded or decreased below certain limits; this would be useful in cases where an apparatus is run without constant attention. 
It is of no value to attempt to determine theoretically the constants of the present voltmeter for purposes of calibration, for the sensitivity is obviously not readily calculated with any considerable accuracy. However, a rough and naive calculation may facilitate the understanding of certain details of the action of the instrument, and guide the selection of dimensions in modified designs. When a potential difference $V$ is maintained between the system $C$ and the can $H$ each ball on the dumb-bell $K$ acquires a charge $q$ and each of the cylinders $L$ is charged; the electrical fields near $K$ and $L$ may be regarded as chiefly due to these charges. Let us consider the repulsion of the charge $q$ in the electric field of the cylinders $L$. It is well known that the force on a very small charge $q$ at a distance $a$ from a long thin wire having a charge $\rho$ per unit length is $2 \rho q / a$. For a rough approximation, we may identify these charges with those on the balls and cylinders of the present instrument and suppose that

$$
F=2 \rho q / a
$$

is the force on one of the balls due to its electrical repulsion by one of the cylinders, where $a$ is the distance from the center of the ball to the axis of the cylinder. Now when the ball is a distance $a$ from one cylinder, it is at a distance

$$
\frac{a}{\tan \theta / 2}
$$

from the other. Accordingly, the repulsion due to this second cylinder is

$$
F \tan \theta / 2
$$

Noticing that the moment arms of these forces about the axis of rotation of the dumb-bell are $(b / 2) \cos \theta / 2$ and $a / 2$, that $a=b \sin \theta / 2$, and remembering that there are two balls and two cylinders, we find for the couple due to the electrical forces

$$
F a\left(\frac{1}{\tan \frac{\theta}{2}}-\tan \frac{\Theta}{2}\right)
$$

For equilibrium, this couple must be balanced against the torsion of the suspension, $k\left(\theta-\Theta_{0}\right)$, where $k$ is a constant for a given suspension and $\Theta_{0}$ is the value of $\theta$ for $V=0$. Thus,

$$
\theta-\Theta_{0}=\frac{F a}{k}\left(\frac{1}{\tan \frac{\theta}{2}}-\tan \frac{\Theta}{2}\right)
$$

Now, in substituting for $F$ in terms of $\rho$ and $q$, it is obvious that all charges on the system $C$ will be proportional to $V$ if the dumb-bell 
is kept at any constant deflection. Accordingly, we may write

where $g$ is a constant. ${ }^{2}$

$$
\rho q=g V^{2} \psi(\Theta)
$$

The factor $\psi(\theta)$ is introduced to allow for the approximations made in assuming infinitely long cylinders, neglecting induction and screening effects, etc. One obvious cause for the variation of $\psi$ with $\theta$ is that as the balls approach the cylinders the charges $q$ and $\rho$ will decrease due to electrostatic induction. Over the range of deflections ordinarily used, however, $\psi$ would not be expected to vary by a large factor. (Compare fig. 3 .) Thus we have finally

$$
\theta-\Theta_{0}=\psi(\Theta) \cdot \frac{2 g}{k}\left(\frac{1}{\tan \frac{\theta}{2}}-\tan \frac{\Theta}{2}\right) \cdot V^{2}
$$

For small deflections, the variation in the multiplier of $V^{2}$ is negligible, so that the deflection, $\theta-\theta_{0}$, is closely proportional to the square of the voltage. For very high voltages, the dumb-bell can never be deflected beyond $\theta=\frac{\pi}{2}$. The maximum possible deflection, $\frac{\pi}{2}-\theta_{0}$, is approached asymtotically as $V$ approaches infinity, and at the same time the sensitivity approaches zero.

The dimensions of the completed instrument were as follows: $R=29 \mathrm{~cm} ; r=1.5 \mathrm{~cm} ; s=1.6 \mathrm{~cm} ; b=9.0 \mathrm{~cm}$. For purposes of test, three different suspensions of tungsten wire were used with diameters and lengths as follows: $0.25 \mathrm{~mm}, 27 \mathrm{~mm}, 0.12 \mathrm{~mm}, 32 \mathrm{~mm}, 0.05 \mathrm{~mm}$, $32 \mathrm{~mm}$. The values of the torsion constants $(k)$ expressed in dyne $\mathrm{cm}$ degree $^{-1}$ were, respectively, 280, 23, and 0.52. These figures, together with the calibration curves of Figure 3 provide all the data necessary for a determination of $\psi$ and its variation with $\theta$; this function $\psi(\theta)$ is shown in the inset of Figure 3. It is to be remembered, of course, that $V$ is expressed in electrostatic units in the formulas. At the higher voltages, an audible brush discharge began. The voltage at which this occurred depended on $\theta$; thus with the various suspensions, $0.25 \mathrm{~mm}, 0.12 \mathrm{~mm}, 0.05 \mathrm{~mm}$, it began at about 95,80 , and $60 \mathrm{kv}$; respectively. This variation is of course connected with the variation with $\theta$ of the electric fields near the balls and cylinders, as indicated by the function $\psi(\Theta)$.

2 The order of magnitude of $g$ may be estimated as follows: Imagine the entire system $C$ replaced by a metal sphere of radius $P$. The charge $\sigma$ per unit area on this sphere would be, with fair approximation, since there would be no other charged bodies inside $H$,

$$
\frac{V R}{4 \pi P(R-P)}
$$

where $R$ is the radius of the can $H$ and $V$ is the difference of potential between $H$ and $C$. Now the system of conductors actually composing $C$ may be thought to approximate, electrically, a spherical network of radius $P$ equal to about $10 \mathrm{~cm}$ in the present construction. Thus the surface charge densities on the outward-facing portions of the cylinders and balls will be roughtly equal to $\sigma$, or allowing for the electrical shielding of inward-facing surfaces, we may suppose the effective charge over the surfaces of balls and cylinders to be of the order of $\sigma / 2$. Accordingly, taking surface areas into account, $q=4 \pi s^{2} \cdot(\sigma / 2)$ and $\rho=2 \pi \tau \cdot(\sigma / 2)$, where 8 and $r$ are the radii of balls and cylinders, respectively. We thus find the order of magnitude of $g$ to be

$$
\frac{r s^{2} R^{2}}{8 P^{2}(R-P)^{2}}
$$


It is of interest to note that formula (1) is of the form

$$
\frac{V}{\sqrt{k}}=f\left(\Theta, \Theta_{0}, R, P, s, r\right)
$$

This shows that, for a given instrument, and for a given zero setting, $\Theta_{0}$, the characteristics are completely described by a function of $V / \sqrt{k}$. Thus only one of the three calibration curves of Figure 3 is required (provided it extends to a sufficient range of angles); all other curves, for various suspensions can immediately be obtained from the one, by multiplying the scale of abscissas by a factor equal to the ratio of the square roots of the torsion constants. This relation may be of value in selecting a suitable suspension; the adjust-

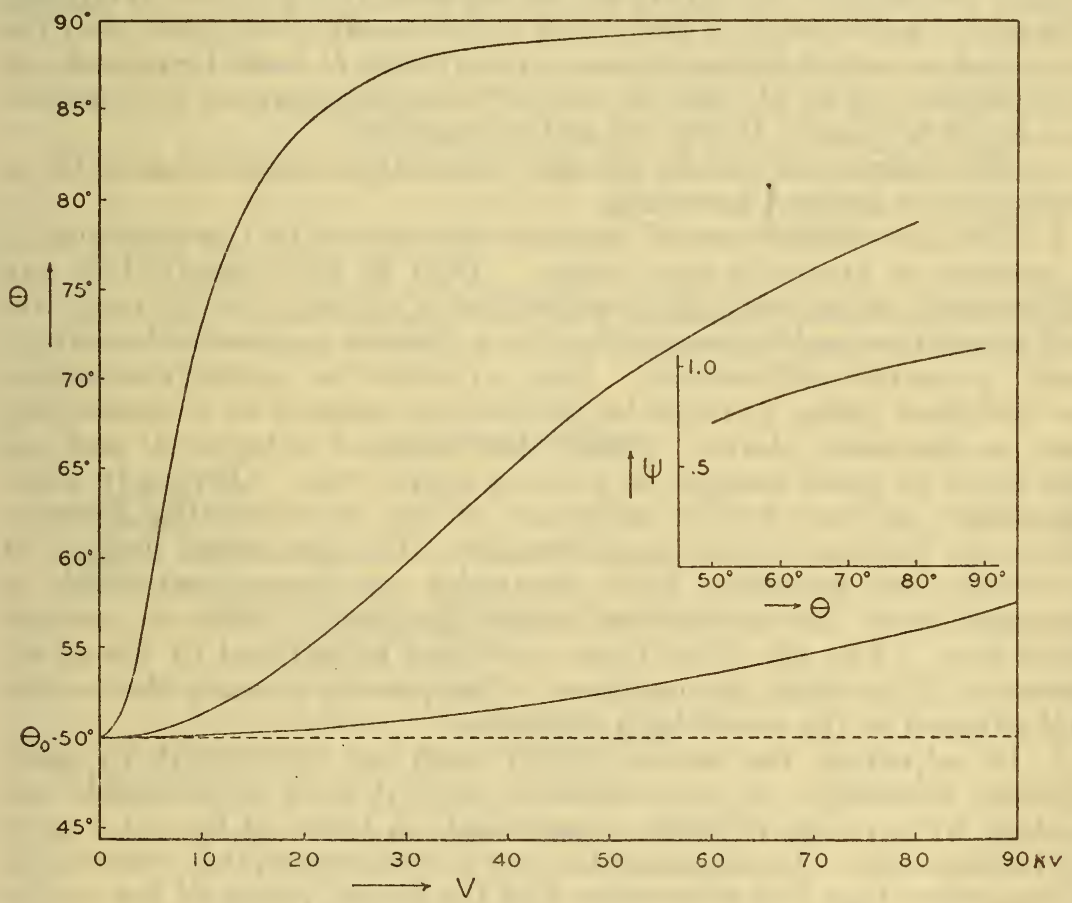

Figdre 3.-Calibration curves for various suspensions.

ment of $\theta_{0}$ is not ordinarily of sufficient precision to make the relation of much value in the final calibration.

The calibration of the present instrument was carried out by means of the high resistance described by Taylor. ${ }^{3}$ If an especially accurate calibration is required, or if the calibration is made by a method less accurate than that used here, the data may be smoothed by any of several methods. One of these methods is to carry out the calibration in $1 \mathrm{kv}$ steps and take first and second differences between the successive values of the deflection. Irregularities will usually appear in the second differences; these irregularities can be made to disappear by a slight adjustment of the data. Another method is to

L. S. Taylor, B. S. Jour. Research, vol. 5 (RP217), p. 609; 1930. 
approximate the data with some readily calculable function; if the approximation is close enough over a moderate range of voltage, a plot of the differences between observed and calculated values will reveal irregularities. These irregularities can then be smoothed out by graphical methods.

In conclusion, some of the advantages of the present instrument are:

1. Only one insulating bushing is required in the construction.

2. The location of the mirror and damping system in the lower end of the instrument is convenient in several ways: It permits the mounting of the voltmeter at a considerable height, which is desirable for safety and for conservation of floor space. Also the mirror and oil are in a location convenient for adjustment without removing $H$ (the viscosity of the oil should be adjusted for optimum damping). Obviously, however, if in some case it were more convenient that the mirror be on top of the instrument, the system $C$ could be introduced through the top of $H$, and the mirror, damping system, and suspension could be placed at the top of the bushing.

3 . The deflections can be readily transmitted mechanically to an apparatus at ground potential.

4. The size of this type of instrument seems to be the minimum it is possible to attain in any design. That is, it is essential in any electrostatic voltmeter to have some kind of system $C$ which measures field strengths (and consequently, for a definite geometrical arrangement, potential differences). Also, in order to properly establish the electrical fields, $C$ must be completely inclosed in a conducting case, as discussed above. Finally the distance between $C$ and the case must be great enough to prevent spark over. Obviously these conditions are met with a minimum of size by concentric spheres, which the present system approximates. In some other designs of voltmeter, for example, both electrodes are introduced inside a grounded case; this necessitates larger distances in order to prevent spark over. The size of all these types can be reduced by use of air pressures higher than atmospheric. The present construction seems well adapted to the use of high pressures.

5. In adjusting the sensitivity of such an instrument to meet required conditions of scale distance, etc., it is of considerable advantage to have the deflections calculable in terms of $\theta_{0}$ and $k$ as is the present case. In adjusting the size of suspension, it is convenient to remember that $k$ is proportional to the fourth power of the radius of the wire used as suspension, and to the inverse square of the period of oscillation of a given suspended system.

Washington, October 15, 1931. 